\title{
Secure E-Health: Managing Risks to Patient Personal Identity
}

\author{
Haryadi Amran D. \\ Mike Yuliana \\ Reni Soelistijorini \\ Amang Sudarsono
}

Electronic Engineering Polytechnic Institute of Surabaya, Indonesia

doi: 10.19044/esj.2017.v13n24p377 URL:http://dx.doi.org/10.19044/esj.2017.v13n24p377

\begin{abstract}
As we are becoming a digital society, it is very important for us to protect the security of personal identity in any transactions. One of the efforts to maintain the security of personal identity is by unrevealed our data more than necessary. Anonymous credential allows an organization to give a credential to a user. This credential consists of user attribute such as address and date of birth. By using this credential, the user can prove the ownership to the third party without revealing the information that contained in credential information more than necessary. This paper proposes secure ehealth system that focuses on proving protocol to reveal and prove some of the patient attributes while others remain hidden, so the security of personal identity more awake. The experimental results showed that the computational time of each proving protocol less than 1 second.
\end{abstract}

Keywords: Identity, anonymous, credential, attribute, e-health

\section{Introduction}

Currently, there are many using the Internet to access a variety of information about e-commerce, e-banking, and e-health, so the number of electronic transactions is increasing. One of the efforts to secure transactions is authentication by a variety of media including mobile phone, RFID tags, and also electronic identity card. The authentication mechanism requires the information of user's personal identity, and this leads to reduced privacy of users because the transactions can be linked. Lack of privacy is a big problem in the case of trust of the user.

Anonymous credential is a technology that allows an organization to give a credential to a user (Bichsel et.al, 2009). This credential consists of user attribute such as address and date of birth. By using this credential, the 
user can prove the ownership to the third party without revealing the information that contained in credential information more than necessary (Yuliana et.al, 2014). For instance, the user can prove the ownership of a credential that contains the user aged over 21 by using an ID card, by not revealing other data unnecessary. This is the reason why anonymous credential becoming very popular because the system can minimize the misuse of data by parties who are not responsible.

There are many types of research about anonymous credential with various methods or algorithms (Yuliana et.al, 2015). In addition to the basic functions of an anonymous credential, there is some research on the development of anonymous credentials such as enhancement of proving attributes (Belenky et al, 2009), an efficiency of coding attributes (Sudarsono et al, 2011), and implementation on Java card (Camenisch et al, 2008). However, that research only focuses on the computational complexity of each scheme, rarely that focus on the implementation and performance of the system.

In this research, it will be proposed secure e-health system that can improve the security of personal identity using anonymous credential. This system allows the user to reveal and prove some of the attributes while others remain hidden not only using CL signature but also by logical relations proof that includes AND, OR, NOT relations on several attributes, so the security of personal identity more awake.

We structure the remainder of this paper as follows. In section 2, related works of personal identity are described, and logic relation proof will be explained. In section 3, we describe secure e-health system design. The proposed secure e-health system will be discussed in section 4. A prototype implementation and performance measurement will be tested at section 5 . Finally, we conclude our paper at section 6.

\section{Related Works}

Some research discusses the authentication system to secure personal data (Maji et al, 2011), and also anonymous authentication in e-health system which satisfies patient's personal information protection (i.e., health status information) from other patients, doctors, or pharmacists using Anonymous Credential System based CL signature and construct the system by Java programming language (Yuliana et.al, 2014).

The other authors extend the anonymous credential such that selection of attributes disclosure becomes more efficient by encoding attributes as prime numbers (Guo et al, 2013). This method provides significant advantages for a device that has limited computing capabilities such as smart card. Some other research also discusses the efficiency of attributes, thereby reducing computational complexity (Guo et al, 2014). 
Anonymous credential system allows users to prove the ownership of the credential, by minimizing the disclosure of information. Some research extends proving protocol of anonymous credential based CL-signature with logic relation proof that includes AND, OR and NOT (Guo et al, 2014). The results show the complexity of computational is constantly based on the amount of exponentiation and pairing. Other researchers discuss security in e-health systems that focused on the security of e commerce, not personal identity (Androulaki et al, 2011).

\section{Logic Relations Proof}

Anonymous system consists of several rules for issuers, recipients, users, and verifiers. On issuing protocol, credential created by the issuers, and recipients will prove the ownership of credential by using proving protocol to the verifiers. Assumed users obtain CL credential that consists of $E$, for example, $\operatorname{signature}(A, e, v)$ on the message $m_{0}$ and $m_{1}$ where $m_{1}=E$.

\section{AND Relation}

Users convince the verifier that credential contains an attribute with a specific value by using AND relation. Assumed attribute encode with prime $e_{j}$, proving that can be done to convince the verifier that $e_{j}$ part of $E$ is:

$$
\begin{aligned}
& P K\left\{\left(\varepsilon, v^{\prime}, \mu_{0}, \mu_{1}^{\prime}\right):\right. \\
& Z \equiv \pm R_{0}^{\mu_{0}}\left(R_{1}^{e_{j}}\right)^{\mu_{1}^{\prime}} A^{\prime} S^{v^{\prime}}(\bmod n) \wedge \\
& \mu_{0} \epsilon \pm\{0,1\}^{l_{m}} \wedge \mu_{1}^{\prime} \epsilon \pm\{0,1\}^{l_{m}-l_{t}} \wedge \\
& \left.\varepsilon \in\left[2^{l_{e}-1}+1,2^{l_{e}}-1\right]\right\}
\end{aligned}
$$

$n$ is 2 primes and $\mathrm{t}$ to be worth \pm 1 . If $Z \equiv \pm R_{0}^{\mu_{0}} R_{1}^{E} A^{\prime \varepsilon} S^{v^{\prime}}$ then $R_{1}^{E}=$ $\left(R_{1}^{e_{j}}\right)^{\mu_{1}^{\prime}}$ and $E=e_{1} \mu_{1}^{\prime}\left(\bmod p^{\prime} q^{\prime}\right)$, so $E=e_{j} \mu_{1}^{\prime}$ to be worth integer and $e_{j}$ is a factor of $E$.

If there are some attributes that encode with primes $e_{i}, e_{j}, e_{l}$ then proving that can be done to convince the verifier $e_{i}, e_{j}, e_{l}$ part of $E$ is :

$$
\begin{aligned}
P K\left\{\left(\varepsilon, v^{\prime}, \mu_{0}, \mu_{1}^{\prime}\right):\right. & \\
Z & \equiv \pm R_{0}^{\mu_{0}}\left(R_{1}^{e_{i} e_{j} e_{l}}\right)^{\mu_{1}^{\prime}} A^{\prime} S^{v^{\prime}}(\bmod n) \wedge \\
& \mu_{0} \in \pm\{0,1\}^{l_{m}} \wedge \mu_{1}^{\prime} \epsilon \pm\{0,1\}^{l_{m}-3 l_{t}} \wedge \\
\varepsilon & \left.\in\left[2^{l^{-}-1}+1,2^{l_{e}}-1\right]\right\}
\end{aligned}
$$

\section{NOT Relation}

Users convince the verifier that credential does not contain an attribute with a specific value by using NOT relation. Proving that $e_{j} \nmid E$ can be done by showing that $a E+b e_{j}=1$, where $a$ and $b$ would not exist if 
$e_{j} \mid E$. After getting a value of $a$ and $b$, users generate random number $r$ and compute commitment $D=g^{E} h^{r} \bmod n$ to be sent to the verifier.

$$
\begin{aligned}
& P K\left\{\left(\varepsilon, v^{\prime}, \mu_{0}, \mu_{1}, \alpha, \beta, \rho, \rho^{\prime}\right):\right. \\
& Z \equiv \pm R_{0}^{\mu_{0}} R_{1}^{\mu_{1}} A^{\prime \varepsilon} S^{v^{\prime}}(\bmod n) \wedge \\
& D \equiv \pm g^{\mu_{1}} h^{\rho} \bmod n \wedge g \equiv \pm D^{\alpha}\left(g^{c_{j}}\right)^{\beta} h^{\rho^{\prime}} \bmod n \wedge \\
& \mu_{0,} \mu_{1} \epsilon \pm\{0,1\}^{l_{m}} \wedge \varepsilon \epsilon\left[2^{l_{e}-1}+1,2^{l_{e}}-1\right]
\end{aligned}
$$

From $D \equiv \pm g^{\mu_{1}} h^{\rho} \bmod n$ and $g \equiv \pm D^{\alpha}\left(g^{e_{j}}\right)^{\beta} h^{\rho^{\prime}} \bmod n$, it could be calculated $g \equiv \pm g^{\mu_{1} \alpha} h^{\rho \alpha}\left(g^{e_{j}}\right)^{\beta} h^{\rho \prime}$. If user calculates $\log _{g} h$ then $\mu_{1} \alpha+$ $e_{j} \beta=1$. $\alpha$ and $\beta$ will exist if $e_{j}$ and $\mu_{1}$ are co-prime so $e_{j} \mid \mu_{1}$. Because $\mu_{1}$ contained in CL signature, then it can be concluded that $e_{j}$ is not one of the attribute that encodes in $\mu_{1}$.

\section{$\underline{\text { OR Relation }}$}

Users convince the verifier that credential contains one of the attributes with a specific value by using OR relation. Proving that credential contains one of the attributes $\left\{e_{1}, \ldots, e_{l}\right\}$ could be done by sending Commitment $D$ to verifier using this protocol:

$$
\begin{aligned}
& P K\left\{\left(\varepsilon, v^{\prime}, \mu_{0}, \mu_{1}, \alpha, \rho, \rho^{\prime}\right):\right. \\
& Z \equiv \pm R_{0}^{\mu_{0}} R_{1}^{\mu_{1}} A^{\prime \varepsilon} S^{v^{\prime}}(\bmod n) \wedge \\
& D \equiv \pm g^{\mu_{1}} h^{\rho} \bmod n \wedge g^{\prod_{i}^{l} e_{i}} \equiv \pm D^{\alpha} h^{\rho^{\prime}} \bmod n \wedge \\
& \mu_{0,} \mu_{1} \epsilon \pm\{0,1\}^{l_{m}} \wedge \varepsilon \epsilon\left[2^{l_{e}-1}+1,2^{l_{e}}-1\right]
\end{aligned}
$$

Credential contained an attribute $e$, so we get the value of $a$ where $a e=\prod_{i}^{l} e_{i}$. If $e$ is not in the list $a$ then $e$ does not divide the product.

The Proposed Secure E-Health System Design

In this section will be proposed a system that can improve the security of personal identity by using anonymous credential system.. This system allows the user to reveal and prove some of the attributes while others remain hidden, so the security of personal identity more awake.

\section{Privacy Requirements}

The ability of the existing system in the hospital to maintain the privacy of personal identity of patients will increase the trust. Doctors only know partial information of the patient's personal identity.

\section{Security Requirements}

There are two types of attributes, namely strong and weak attributes. Strong attributes are unique for each user such as address, and phone number 
while weak attributes could be the same for each user such as name, and blood type. In this system, the strong attributes contain sensitive information that remained hidden when proving while weak attributes will be revealed. Disclosure of weak attributes must fulfill the security requirements where the user can select the attributes that will be revealed to prevent the disclosure of more information than required.

\section{Proof of Attributes Protocol}

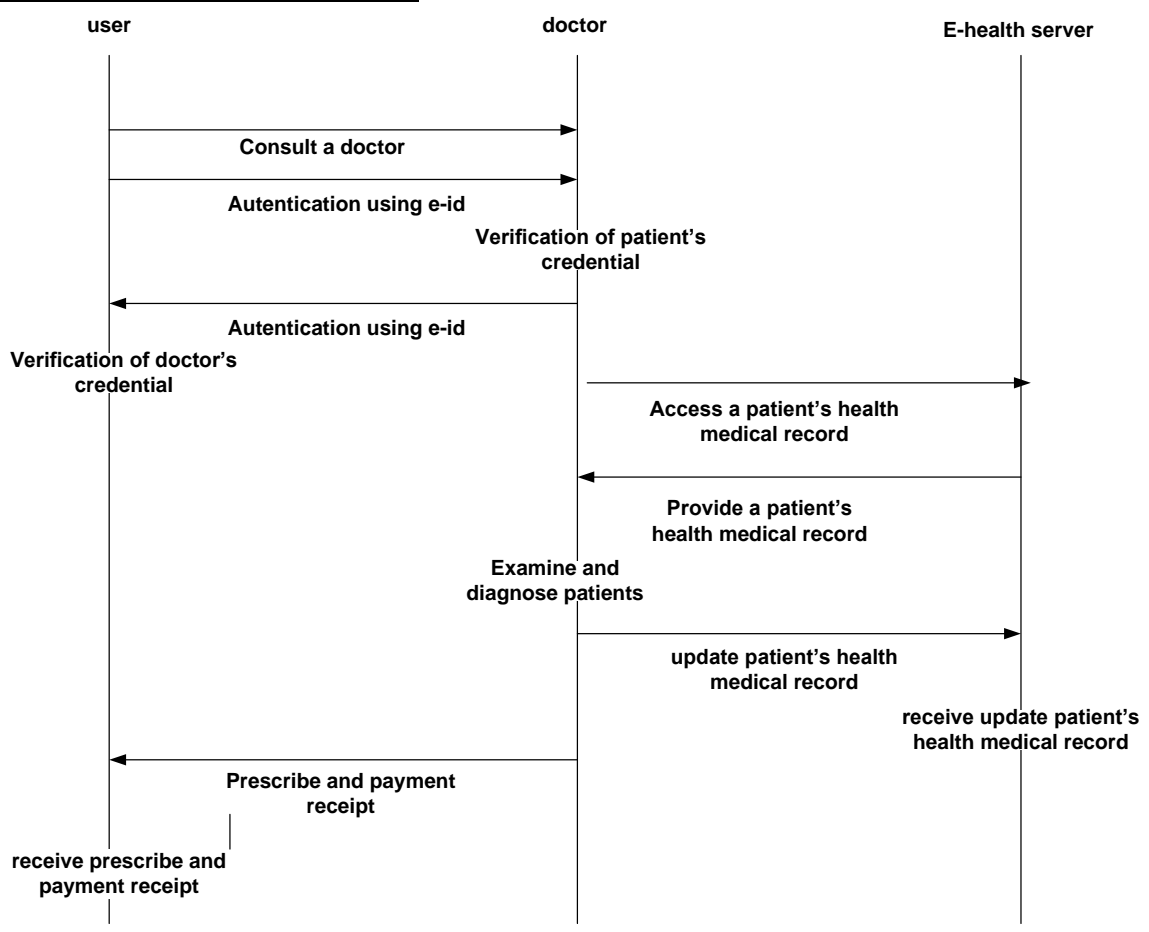

Figure 1. Protocol of consult a doctor.

In this research, a credential from several attributes loaded into e-id, where e-id is obtained during the process of registration and used for the authentication process. Fig. 1 shows the protocol when consulting a doctor. It can be seen that the doctor can see the patient's health medical record if the authentication success performed by doctors and patients.

\section{Prototype Implementation and proof of attributes testing}

In this section will be explained the implementation of the prototype system, the results of testing proving algorithms and system performance. Specifications of Personal Computer can be seen in Table 1 and RFID reader and tag can be seen in Fig. 2 . 
Table 1. Specification of Personal Computer

\begin{tabular}{|c|c|}
\hline Specifications & Remarks \\
\hline Operation System & $\begin{array}{c}\text { Microsoft Windows 7 } \\
\text { Professional 64-Bit (Build 7601) }\end{array}$ \\
\hline Processor & $\begin{array}{c}\text { Intel Core i5-2450M (2.50 GHz, } \\
\text { 3MB L3 Cache) (4 CPUs) }\end{array}$ \\
\hline Memory & 8 GB RAM \\
\hline
\end{tabular}

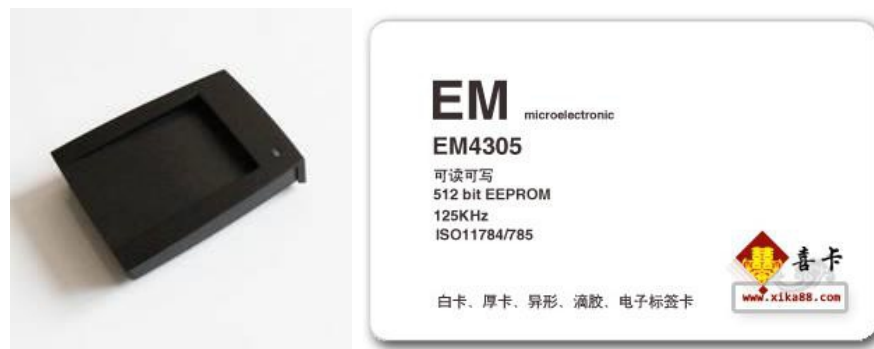

Figure 2. RFID reader and tag

Attributes are information that used during the registration process, in which the attributes information consists of name, issuance modes (known, hidden) and type (int, String, enumeration). Figure 3 shows the registration form, in which the attributes that are used include name, address, phone number, identity card number, sex, birth date, blood type, civil status, and employment. Address, phone number, and identity card number are strong attributes and the others are weak attributes. the issuance mode of strong attributes are hidden and weak attributes are known, so the user that acts as prover knows all the attribute values but the verifier only knows weak attributes.
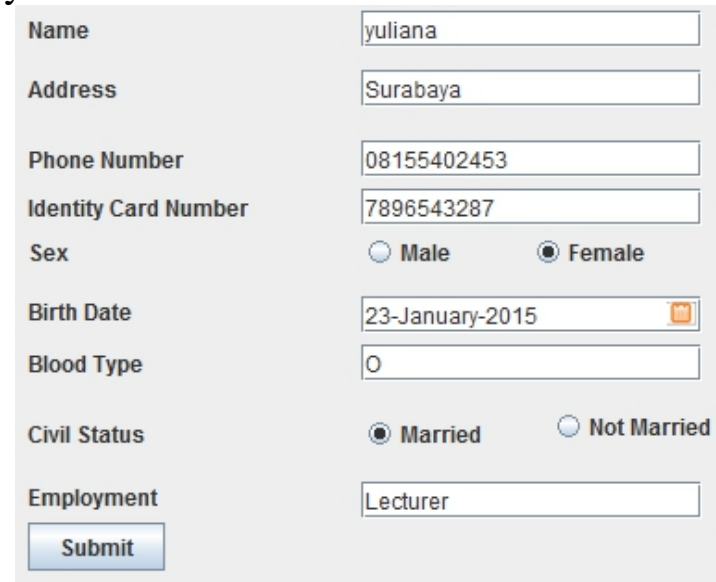

Figure 3. Registration form. 


\section{Proof of Attributes Testing}

In this section will be performed some testing which includes proof of CL signature, proof of AND, OR and NOT relations.

\section{Proof of CL Signature}

In this experimental test, it will be proved that the value of $A, e, v$ are the signature of attributes. Fig. 3 show the example of signature from 9 attributes. The value that will be obtained when authentication and verification process is the success are $t$ (proof process) and $\hat{t}$ (verify process) can be seen in Fig. 5. The test results showed that the proof is not rejected.

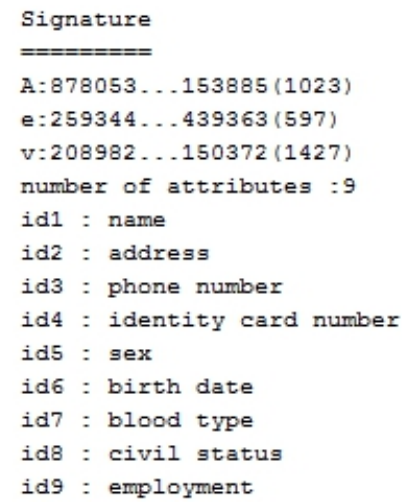

Figure 4. Example of signature

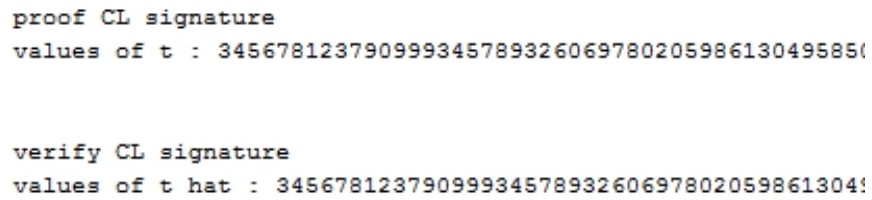

Figure 5. Proof and verify CL Signature

\section{Proof of AND Relation}

In this experimental test, it will be proved that the patient will get medical check fee discount if the patient was a lecturer and not married. The value that will be obtained when authentication and verification process is the success are $\tilde{C}, \tilde{C}_{0}$ (proof process) and $\hat{C}, \hat{C}_{0}$ (verify process) that can be seen in Fig. 6. The test results showed that the proof is not rejected and the patient can get medical check fee discount. 


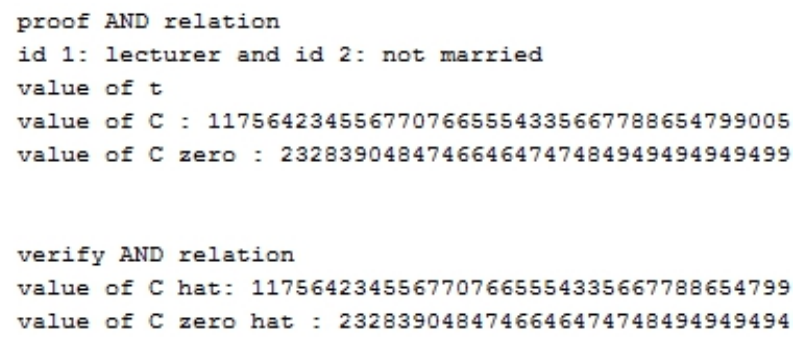

Figure 6. Proof and verify AND relation

\section{Proof of OR Relation}

In this experimental test, it will be proved that the patient will get medical check fee discount if the patient was a lecturer or not married. The value that will be obtained when authentication and verification process success is $\tilde{T}_{1}, \widetilde{T}_{2}, \tilde{T}_{3}, \widetilde{T}_{4}, \tilde{T}_{5}, \widetilde{T}_{6}$ (proof process) and $\widehat{T}_{1}, \widehat{T}_{2}, \widehat{T}_{3}, \widehat{T}_{4}, \widehat{T}_{5}, \widehat{T}_{6}$ (verify process) that can be seen in Fig. 7. The test results showed that the proof is not rejected and the patient can get medical check fee discount.

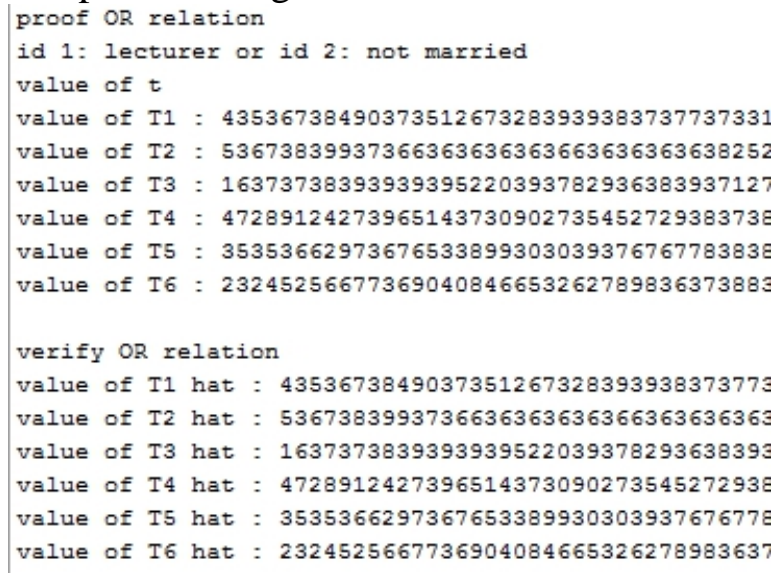

Figure 7. Proof and verify OR relation

\section{Proof of NOT Relation}

In this experimental test, it will be proved that the patient will get medical check fee discount if the patient's civil status not married. The value that will be obtained when authentication and verification process is the success are $\tilde{C}, \tilde{C}_{c}$ (proof process) and $\hat{C}, \hat{C}_{c}$ (verify process) that can be seen in Fig. 8. The test results showed that the proof is not rejected and the patient can get medical check fee discount. 


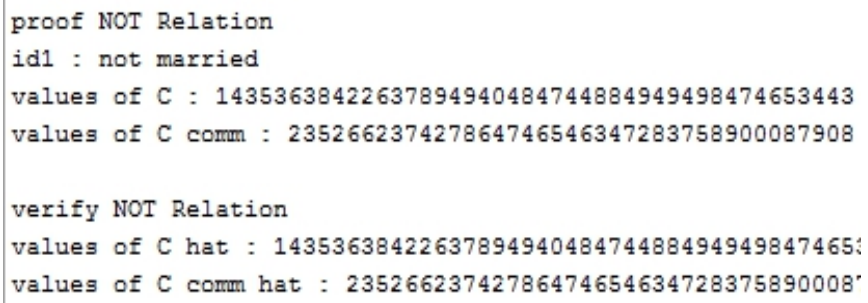

Figure 8. Proof and verify NOT relation

\section{E-Health System Performance}

In this section will be tested computational time for proving protocol. There are 3 kinds of schemes, namely testing for revealed, unrevealed and combination. For proving protocol with all attributes revealed, the attributes have issuance mode known, so when authentication process all of these attributes will be revealed to the verifier. Fig. 9 shows the computational time of proving protocol for 2 through 9 attributes. The test results show that the computational time from 2 through 9 attributes do not have a lot of time difference. The highest computational time is 0.8866 seconds occurs when the number of attributes 9 .

For proving protocol with attributes unrevealed, the attributes have issuance mode hidden, so when authentication process all of these attributes will not be revealed to the verifier. Fig. 10 shows the computational time of proving protocol for 2 through 9 attributes. The test results show that the computational time from 2 through 9 attributes do not have a lot of time difference. The highest computational time is 0.91 seconds occurs when the number of attributes 7 .

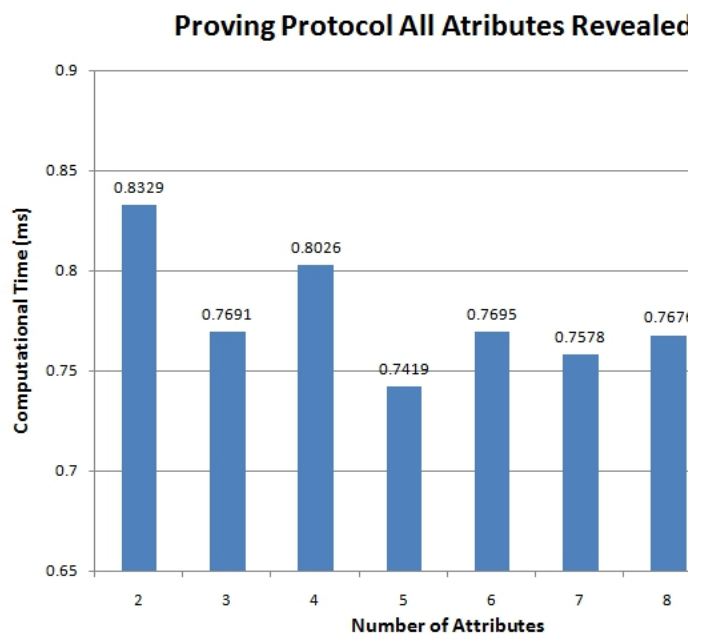

Figure 9. Proving protocol with all attributes revealed 


\section{Proving Protocol All Attributes Unrevealed}

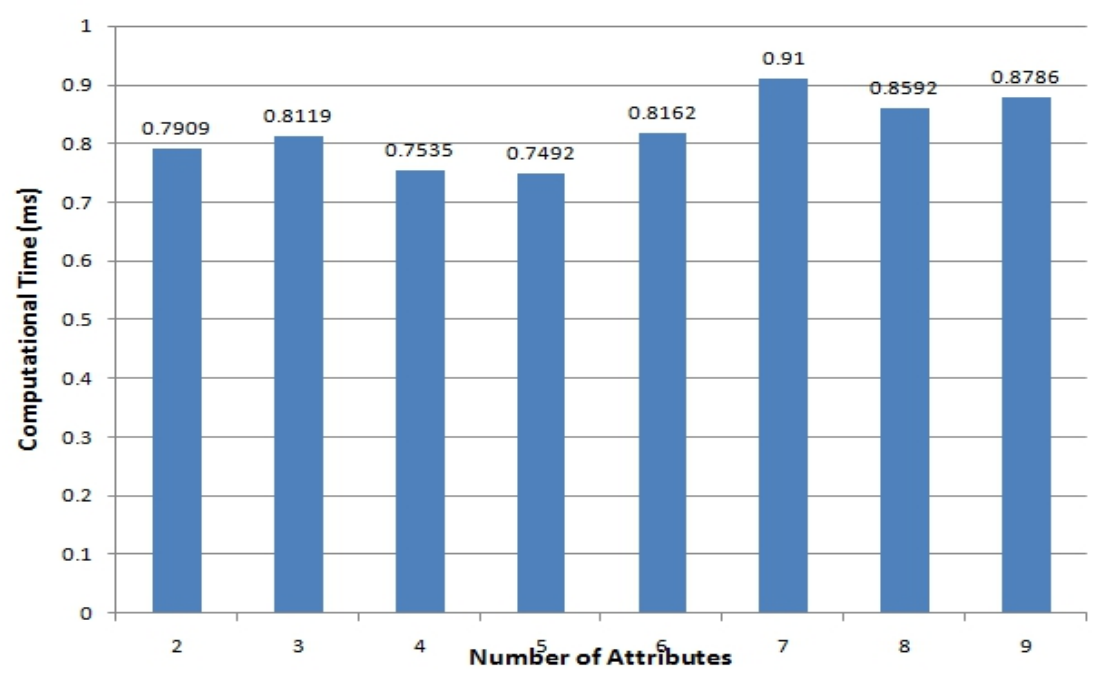

Figure 10. Proving protocol with all attributes unrevealed

For proving protocol with a combination of both attribute, the attributes have issuance mode known and hidden, so when authentication process some of these attributes will be revealed to the verifier and the others remain hidden. Fig. 11 shows the computational time of proving protocol for 2 through 9 attributes. The test results show that the computational time from 2 through 9 attributes do not have a lot of time difference. The highest computational time is 0.8426 seconds occurs when the number of attributes 3.

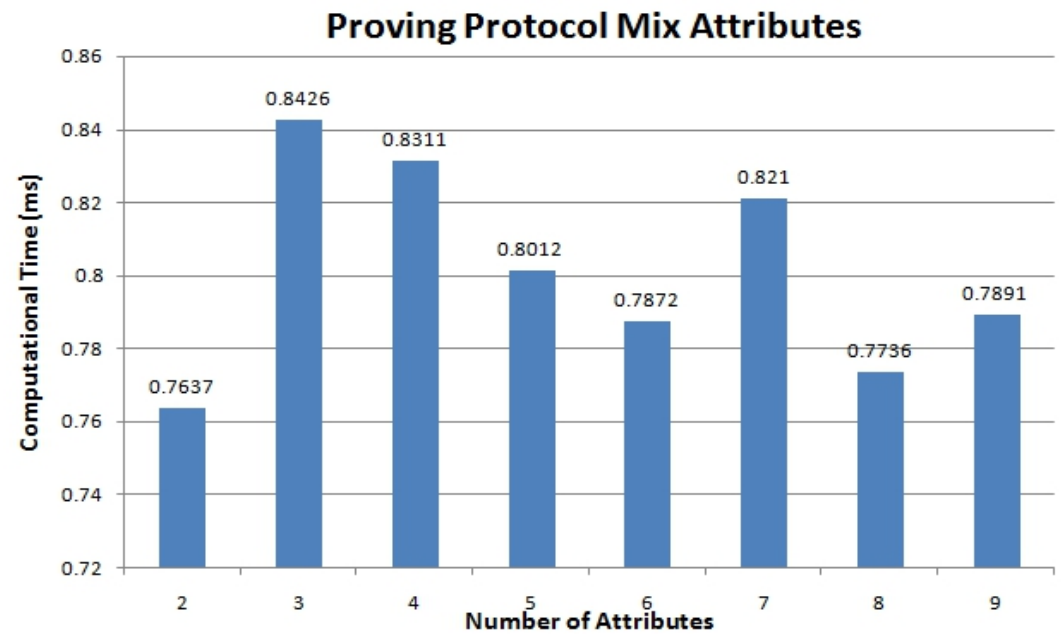

Figure 11. Proving Protocol with mix attributes 
Fig. 12 shows the comparison of computational time from 3 kinds of proving protocol, where the results showed that no significant difference in computational time for different number and types of attributes. The computational time of proving protocol is less than 1 second.

\section{Proving Protocol}

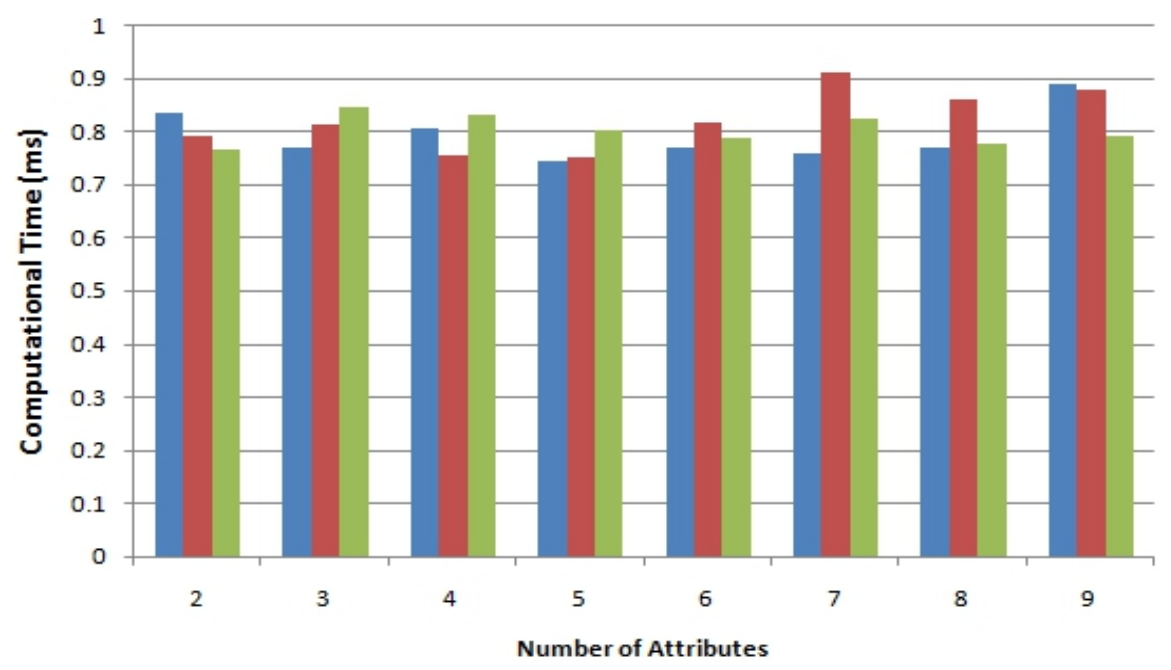

Figure 12. Comparison of 3 kinds of proving protocol.

\section{Conclusion}

We proposed secure e-health system design that can be fulfilled the privacy and security requirements. The system allows the user to reveal and prove some of the attributes while others remain hidden, so the security of personal identity will be more secure. Proof attributes testing includes the selection of attributes disclosure, proof of CL signature, proof of AND, OR and NOT relations. The computational time for each proving protocol is less than 1 second. Overall it can be said that the system designed was able to increase the security of personal identity, thereby reducing the misuse of personal identity by unauthorized parties.

\section{Acknowledgment}

This research was supported by Ministry of Research, Technology, and Higher Education through Penelitian Produk Terapan Scheme 2017.

\section{References:}

1. Bichsel, P., Camenisch,J., Gro, T., \& Shoup,V., Anonymous credentials on a standard java card, in Proc. ACM Conference on 
Computer and Communications Security 2009 (ACM-CCS'09), pp. 600-610, 2009.

2. Yuliana, M., Sudarsono, A. \& Nadhori, U.I, Protection system of client's privacy on e-health services, In Proc. Of the $8^{\text {th }}$ International Conference on Information and Communication Technology and System (ICTS), pp.291-296 , 2014.

3. Yuliana, M., Pratiarso, A. \& Sudarsono, A., Proof of Attributes Based CL Signature Scheme on E-Health Applications, in Proc. Of International Conference on Science in Information Technology (ICSITech), pp. 253-258, 2015.

4. Belenky, M., Camenisch, J. \& Chase, M., Randomizable proofs and delegetable anonymous credentials, in. Proc. Of Crypto, 2009.

5. Sudarsono, A., Nakanishi \& T. \& Funabiki, N., Efficient Attributebased signatures for non-monotone predicates in the standard model, In Proc. Of the $11^{\text {th }}$ International Symposium Privacy Enhancing Technologies (PETS'11),pp.246-263,2011.

6. Camenisch, J. \& Grob, T. Efficient Attributes for Anonymous Credential, in Proc. Of ACM Conference on Computer and Communications Security, pp.345-356, 2008.

7. Maji, H.K.,Prabhakaran \&Rosulek, M., Attribute-based signatures, in Proc.of Cryptographers'Track at the RSA Conference 2011 Topics in Cryptology (CT-RSA'11), pp. 376-392, 2011.

8. Guo, N., Cheng, J., Zhang, B. \& Yim, K., Aggregate signature-based efficient attributes proof with pairing-based anonymous credential, in Proc. Of Network-based Information System (NBiS), pp.276-281, 2013.

9. Guo, N., Wang, J.,Gao, T. \& Yim, K., Privacy-preserving proof af attributes with CL-Anonymous Credential, Journal of Internet Services and Information Security (JISIS), volume:4,number:1,pp. 37-46, 2014.

10. Androulaki, E., A privacy preserving e commerce oriented identity management architecture, PhD Dissertation, Columbia University, New York, 2011. 